\title{
Perineal Pain
}

National Cancer Institute

\section{Source}

National Cancer Institute. Perineal Pain. NCI Thesaurus. Code C78529.

Painful sensation in the perineal area. 